\title{
Numerical modelling of natural convection in a square cavity: effect of nanofluid volume fraction and inclination
}

\author{
M.M. Charafi ${ }^{\mathrm{a}}$, A. Bendaraa ${ }^{\mathrm{b}}$, A. Hasnaoui ${ }^{\mathrm{c}}$ \\ LS3M, Faculté Polydisciplinaire de Khouribga Univ. Sultan My Slimane, 25000 Khouribga, Morocco.
}

\begin{abstract}
In this paper, the phenomenon of natural convection in a square cavity filled with copper $(\mathrm{Cu})$ based nanofluid (Nf) has been studied. The influences of the volume fraction $(\phi)$ on the convective flow, as well as the effect of the inclination $(\gamma)$ of the cavity on thermal performances were examined. The dimensionless governing equations formulated using stream function, vorticity, and temperatures were solved by finite difference methods, where the Successive Over-Relaxation (SOR) iteration and the upwind scheme were adopted. The developed code served as a verification of results where it showed a good agreement with previous works. The calculation code was then used to visualize the influence of the inclination on the thermal enhancement and showed that the transfer becomes more efficient with an inclination of $\pi / 6$
\end{abstract}

\section{Introduction}

The phenomenon of convection remains a current and much diversified research axis. This is justified by a set of recently published research works. The mastery and performance management of this type of transfer has become a major issue in the industry, its study has been the subject of several research projects crowned by publications in the field [1-5]. At the laboratory scale, several works have been focused on the study of convective flows in several geometries, namely square cavities.

Karatas et al. [6] carried out an experimental study of the phenomenon of convection in a rectangular cavity subjected to a radiation considered as a source of thermal excitation. In the same context, convection within a ventilated cavity has been the subject of a numerical work by Ismael el al. [7]. Thus, several numerical methods have been implemented to solve the problem of convection in cavities. Latice Boltzman Methode (LBM) has been used by Haouat et al. [8] to analyze the same problem. Wang et al. [9] have opted for Chebyshev spectral method to analyze convection in a cavity with a porous media.

Other works concentrated on the study of the nanofluids in the square cavities [10]. Boualit et al. [11] and Khanafer et al. [12] used finite element as well as finite volume methods to solve the same problems. Kogawa et al. [13] investigated the influence of the concentration of nanofluids on thermal convection.

The objective of this work is the development of a numerical code to study the phenomenon of convection in a square cavity filled with nanofluids. Thus, the simulation of the natural convection of the fluid under the effect of a temperature gradient, and the study of the

\footnotetext{
a e-mail: mcharafi@hotmail.com

b e-mail: anass.bendaraa@gmail.com

c e-mail: hasnaoui59@hotmail.com
}

effect of the nanoparticle volume fraction on the improvement of the heat transfer will allow the validation of the code, by comparing the present results with previous works. The code will then be used to analyze the effect of cavity inclination on thermal performance.

\section{Mathematical formulation}

The studied configuration is a square cavity with rigid walls, filled with a nanofluid. This configuration is subjected to a temperature gradient with the hot temperature (Th) imposed on the left wall while the cold temperature (Tc) is on the right wall (Dirichlet conditions). The other walls are adiabatic as it is shown in the schema presented in Fig.1.

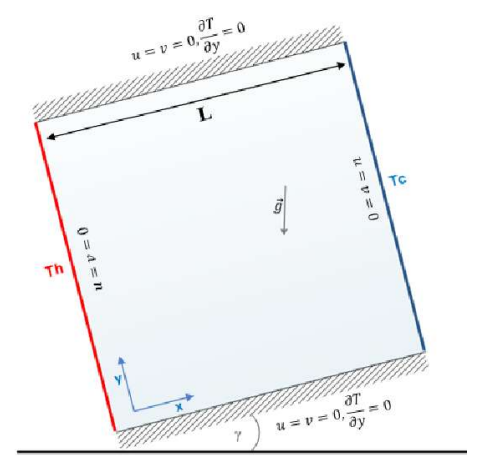

Fig. 1. Schematic representation for the physical model.

The used nanofluid is based on a suspension of copper $(\mathrm{Cu})$ nanoparticles. The fluid flow in the cavity is assumed to be Newtonian and incompressible. The 
governing equations formulated using stream function, vorticity, and temperatures are expressed by the following system of equations:

$$
\begin{gathered}
\frac{\partial \omega}{\partial t}+u \frac{\partial \omega}{\partial x}+v \frac{\partial \omega}{\partial y}=\frac{\mu_{e f f}}{\rho_{n f}}\left(\frac{\partial^{2} \omega}{\partial x^{2}}+\frac{\partial^{2} \omega}{\partial y^{2}}\right)+ \\
\frac{g}{\rho_{n f}}\left[\phi \rho_{s} \beta_{s}+(1-\phi) \rho_{f} \beta_{f}\right]\left[\cos (\gamma) \frac{\partial T}{\partial x}-\sin (\gamma) \frac{\partial T}{\partial y}\right] \\
\frac{\partial T}{\partial t}+u \frac{\partial T}{\partial x}+v \frac{\partial T}{\partial y}=\frac{\partial}{\partial x}\left[\left(\alpha_{n f}+\frac{k_{d}}{\left(\rho c_{p}\right)_{n f}}\right) \frac{\partial T}{\partial x}\right]+ \\
\frac{\partial}{\partial y}\left[\left(\alpha_{n f}+\frac{k_{d}}{\left(\rho c_{p}\right)_{n f}}\right) \frac{\partial T}{\partial y}\right] \\
\frac{\partial^{2} \psi}{\partial x^{2}}+\frac{\partial^{2} \psi}{\partial y^{2}}=-\omega
\end{gathered}
$$

With the following initial and boundary conditions:

$$
\begin{gathered}
u=v=T=0 \text { at } t=0 \text { every where } \\
\left\{\begin{array}{cll}
\text { at } t>0 \\
u=v=\frac{\partial T}{\partial y}=0 & \text { for } y=0, \mathrm{~L} \text { and } 0 \leq x \leq L \\
T=T_{h}, u=v=0 & \text { for } x=0 & \text { and } 0 \leq y \leq L \\
T=T_{c}, u=v=0 & \text { for } y=L & \text { and } 0 \leq x \leq L
\end{array}\right.
\end{gathered}
$$

Here, $\mathrm{x}$ and $\mathrm{y}$ are spatial coordinates, $\mathrm{u}$ and $\mathrm{v}$ are the velocities components, $T$ is the temperature, $\alpha_{n f}$ the nanofluid thermal diffusivity, $\mu_{\text {eff }}$ is the effective dynamic viscosity, $\rho_{\mathrm{nf}}, \rho_{\mathrm{s}}$ and $\rho_{\mathrm{f}}$ are respectively the densities of nanofluid, nanoparticles and the density of fluid, $\beta_{\mathrm{nf}}, \beta_{\mathrm{f}}$ and $\beta_{\mathrm{s}}$ are respectively the thermal expansion of nanofluid fluid and nanoparticles, $\phi$ is the volume fraction, $\mathrm{k}_{\mathrm{d}}$ is the thermal conductivity of the dispersion, and $\left(\mathrm{k}_{\text {eff }}\right)_{\text {stagnant }}$ is the effective stagnant conductivity [12], $\mathrm{C}_{\mathrm{p}}$ the specific heat, the constant $\mathrm{C}$ is determined experimentally $\mathrm{C}=0.4 \quad[11-12]$ for cooper-water nanofluid, $\psi$ is the stream function and $\omega$ is the vorticity.

To obtain the dimensionless equations, we introduce the dimensionless spatial variables undermentioned:

$$
\left.\begin{array}{c}
X=\frac{x}{L}, Y=\frac{y}{L}, U=\frac{u}{\sqrt{g \beta_{f} \Delta T L}}, V=\frac{v}{\sqrt{g \beta_{f} \Delta T L}} \\
\tau=\frac{t \sqrt{g \beta_{f} \Delta T L}}{L}, \Omega=\frac{\omega L}{\sqrt{g \beta_{f} \Delta T L}}, \Psi=\frac{\psi}{L \sqrt{g \beta_{f} \Delta T L}}, \theta=\frac{T-T_{c}}{T_{h}-T_{c}}
\end{array}\right\}
$$

The system of dimensionless equations will be written as follows:

$$
\begin{aligned}
& \frac{\partial \Omega}{\partial \tau}+U \frac{\partial \Omega}{\partial X}+V \frac{\partial \Omega}{\partial Y}=\frac{\left(\frac{\partial^{2} \Omega}{\partial X^{2}}+\frac{\partial^{2} \Omega}{\partial Y^{2}}\right)}{(1-\phi)^{2.5}\left[\phi \frac{\rho_{s}}{\rho_{f}}+(1-\phi)\right] \sqrt{G r}}+ \\
& \lambda\left[\cos (\gamma) \frac{\partial \theta}{\partial X}-\sin (\gamma) \frac{\partial \theta}{\partial Y}\right],
\end{aligned}
$$

$$
\begin{aligned}
\frac{\partial \theta}{\partial \tau}+U \frac{\partial \theta}{\partial X}+V \frac{\partial \theta}{\partial Y}= & \frac{1}{\operatorname{Pr} \sqrt{G r}}\left[\frac{\partial}{\partial X}\left(\chi \frac{\partial \theta}{\partial X}\right)+\frac{\partial}{\partial Y}\left(\chi \frac{\partial \theta}{\partial Y}\right)\right], \\
& \frac{\partial^{2} \Psi}{\partial X^{2}}+\frac{\partial^{2} \Psi}{\partial Y^{2}}=-\Omega
\end{aligned}
$$

With:

$$
\chi=\frac{\left[\frac{\left(k_{\text {eff }}\right)_{\text {stagnant }}}{k_{f}}\right]}{(1-\phi)+\phi \frac{\left(\rho C_{p}\right)_{s}}{\left(\rho C_{p}\right)_{f}}}+C \phi \frac{d_{p}}{L} \operatorname{Pr} \sqrt{G r} \sqrt{U^{2}+V^{2}}
$$

And:

$$
\frac{\left(k_{\text {eff }}\right)_{\text {stagnant }}}{k_{f}}=\frac{k_{s}+2 k_{f}-2 \phi\left(k_{f}-k_{s}\right)}{k_{s}+2 k_{f}+2 \phi\left(k_{f}-k_{s}\right)}
$$

In this work, the particle diameter used is $d_{p}=10 \mathrm{~nm}$, and the characteristic dimension of the cavity "L" is taken equal to $1 \mathrm{~cm}$,

The dimensionless numbers are expressed using the thermophysical properties of the base fluid. The Prandtl number (Pr) and the Grashof number (Gr) as well as the coefficient $\lambda$ which appears in the term of thermal expansion are given by:

$$
\operatorname{Pr}=v_{f} / \alpha_{f}, \quad G r=g \beta_{f} \Delta T L^{3} / v_{f}^{2}, \quad \lambda=\frac{\beta_{n f}}{\beta_{f}}
$$

In order to quantify the thermal transfer within the cavity, and to characterize the effect of increasing nanofluid volume fraction, the value of the local Nusselt and the Nusselt average must be determined. These parameters are calculated as follows:

$$
N u=-\left.\frac{k_{n f}}{k_{f}} \frac{\partial \theta}{\partial X}\right|_{X=0}, N u_{\text {aver }}=\int_{0}^{1} N u(Y) \mathrm{dY}
$$

\section{Numerical method}

The governing Eqs. (6)-(8) with corresponding initial and boundary conditions have been solved by finite difference method, where the Successive OverRelaxation (SOR) iteration is used for the stream function equation (8) and the Upwind scheme is used for vorticity and temperature equation (6-7). The convergence criterion used is: $\frac{\sum\left|A_{i, j}^{n+1}-A_{i, j}^{n}\right|}{\sum\left|A_{i, j}^{n+1}\right|}<10^{-6}$ where the parameters A represents, the stream function $\Psi$, the temperature $\theta$ or the vorticity $\Omega$.

Vorticities at the boundaries are found from Taylor's developments of the stream functions, and are be given as follows:

$$
\left\{\begin{array}{l}
\Omega_{i, 1}=-2 \frac{\Psi_{i, 2}}{(\Delta X)^{2}}, \Omega_{i, n x}=-2 \frac{\Psi_{i, n y-1}}{(\Delta X)^{2}} \\
\Omega_{1, j}=-2 \frac{\Psi_{2, j}}{(\Delta Y)^{2}}, \Omega=-2 \frac{\Psi_{n x-1, j}}{(\Delta Y)^{2}}
\end{array}\right.
$$




\section{Volume fraction effects}

To validate the developed code, a series of numerical experiments carried out to describe the flow profiles into the cavity with $\gamma=0^{\circ}$. These experiments are done for several mesh spacing from $41 \times 41$, to $91 \times 91$. We choose the spacing $81 \times 81$ for his better ratio between simulation time and accuracy of the results. These results are compared with those of Khanafer et al. [12]. In addition to the good agreement with the results of Khanafer et al. [12], the influence of the volume fraction on the streamlines and isotherms is shown in Figs. 2-5. These figures illustrate the effects of the volume fraction on the isothermal lines and the stream lines for a number of $\mathrm{Gr}=10^{4}$. The analysis of these figures shows that the increase of the nanoparticle volume fraction leads to an intensification of the central vortices, which tends to become elliptical. These results can be explained by the influence of the volume fraction of the nanoparticles on the viscous forces and the buoyant forces interacting during a natural convection flow.

Khanafer et al. results

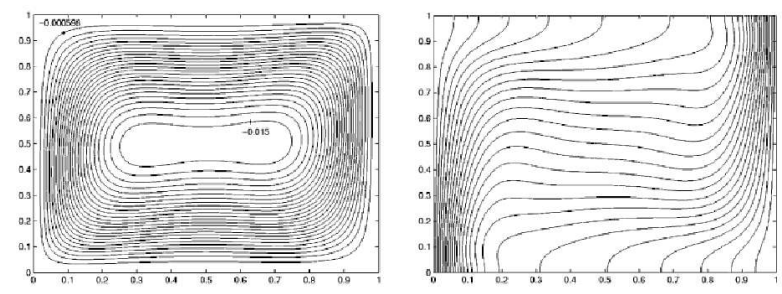

Present work
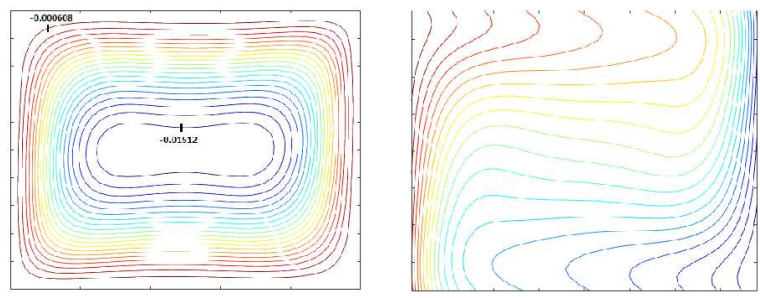

Fig. 2. Comparison of the streamlines and the isotherms between the present work and [12] $\left(\mathrm{Pr}=6.2, \mathrm{Gr}=10^{4}, \phi=0 \%\right.$, $\left.\gamma=0^{\circ}\right)$.

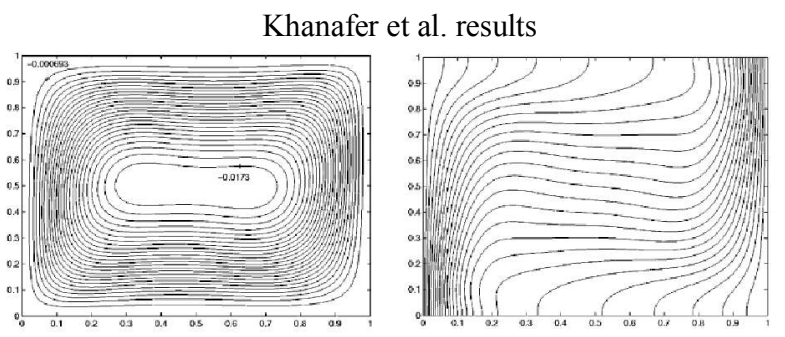

Present work
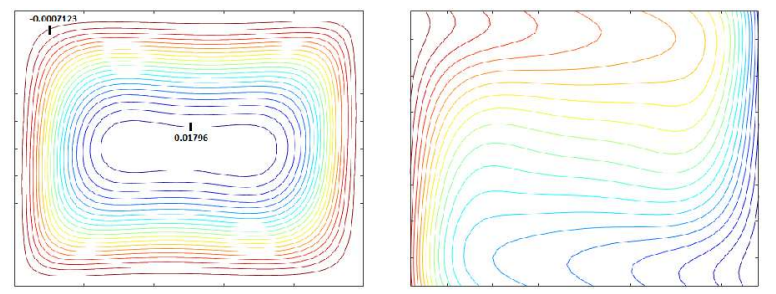

Fig. 3. Comparison of the streamlines and the isotherms between the present work and [12] $\left(\mathrm{Pr}=6.2, \mathrm{Gr}=10^{4}, \phi=8 \%\right.$, $\gamma=0^{\circ}$ ).

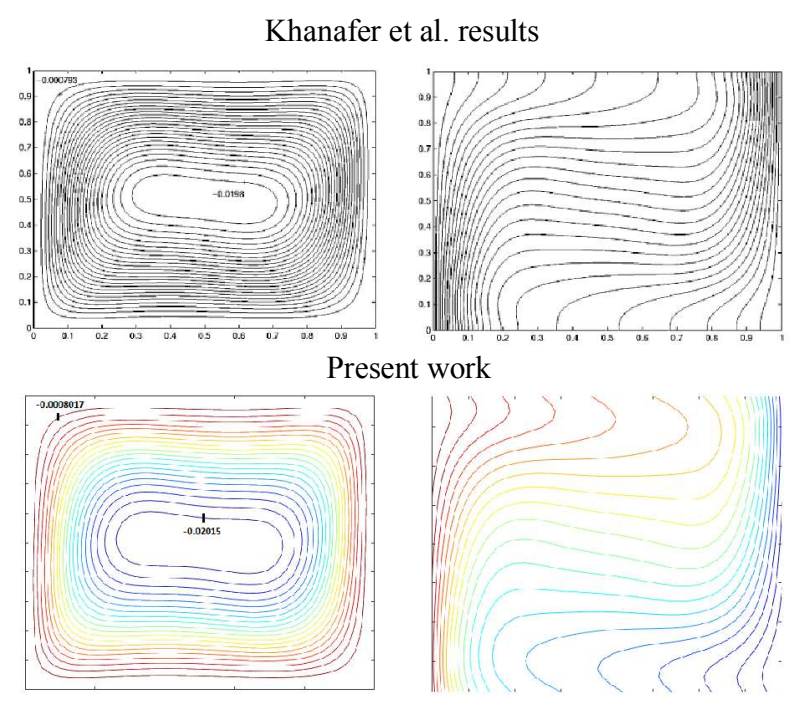

Fig. 4. Comparison of the streamlines and the isotherms between the present work and [12] $\left(\mathrm{Pr}=6.2, \mathrm{Gr}=10^{4}, \phi=16 \%\right.$, $\gamma=0^{\circ}$ ).

Khanafer et al. results
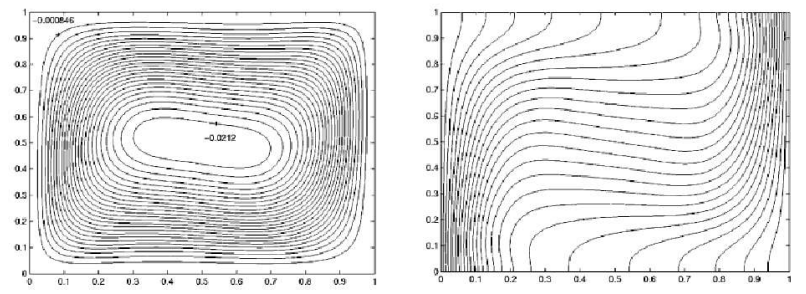

Present work
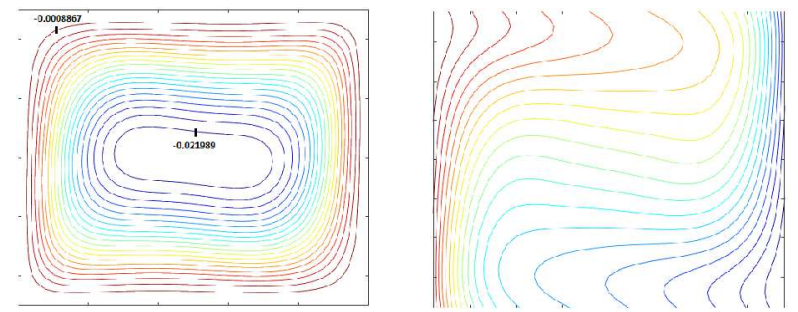

Fig. 5. Comparison of the streamlines and the isotherms between the present work and [12] $\left(\operatorname{Pr}=6.2, \mathrm{Gr}=10^{4}, \phi=20 \%\right.$, $\gamma=0^{\circ}$ ).

A comparison of the Nusselt values, between the present work and Khanafer et al. [12] is presented in Fig. 6-a. This figure shows a good agreement between the results. It can be also seen that the transfer intensifies by increasing the volume fraction that is explained by the $\mathrm{Nu}$ increasing.

\section{Inclination effects}

In this section, after testing the calculation code, which gave a good agreement with result of Khanafer et al. [12], the influence of the inclination of the cavity on the nanofluid flow, as well as, on the thermal performances are investigated, where a series of simulations are performed. A cooper $(\mathrm{Cu})$ nanoparticle volume fraction of $10 \%$ is used with different inclinations ranging from $0^{\circ}$ to $\pi / 2$ and for values of Grashof number ranging from $10^{3}$ to $10^{5}$. 

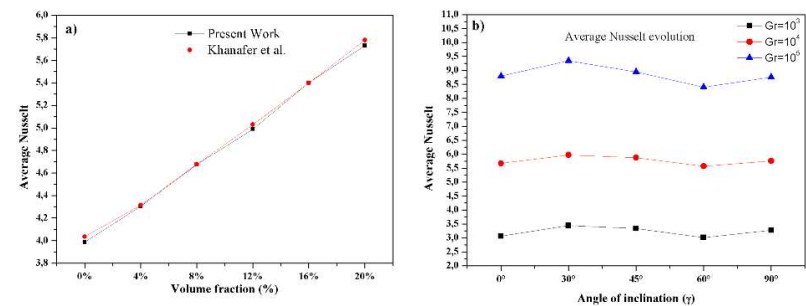

Fig. 6. a) The comparison of Nusselt between the present work \& Khanafer $\left(\operatorname{Pr}=6.2, \gamma=0^{\circ}\right)$. b) Evolution of the Nusselt number as a function of the inclination angle for different $\mathbf{G r}$ numbers $(\operatorname{Pr}=6.2, \phi=0 \%)$.

Figure 6-b illustrates the evolution of the average number of Nusselt in the vicinity of the hot wall, thus allowing quantifying the rate of improvement of the heat transfer by convection in the cavity. As shown in Fig. 6, the heat transfer becomes more intense by increasing the number of Grashof (Gr). On the other hand, the inclination of the cavity has an influence on the number of Nusselt and marks a maximum for an inclination of $\pi / 6$ and a minimum of performance at an inclination of $\pi / 3$.

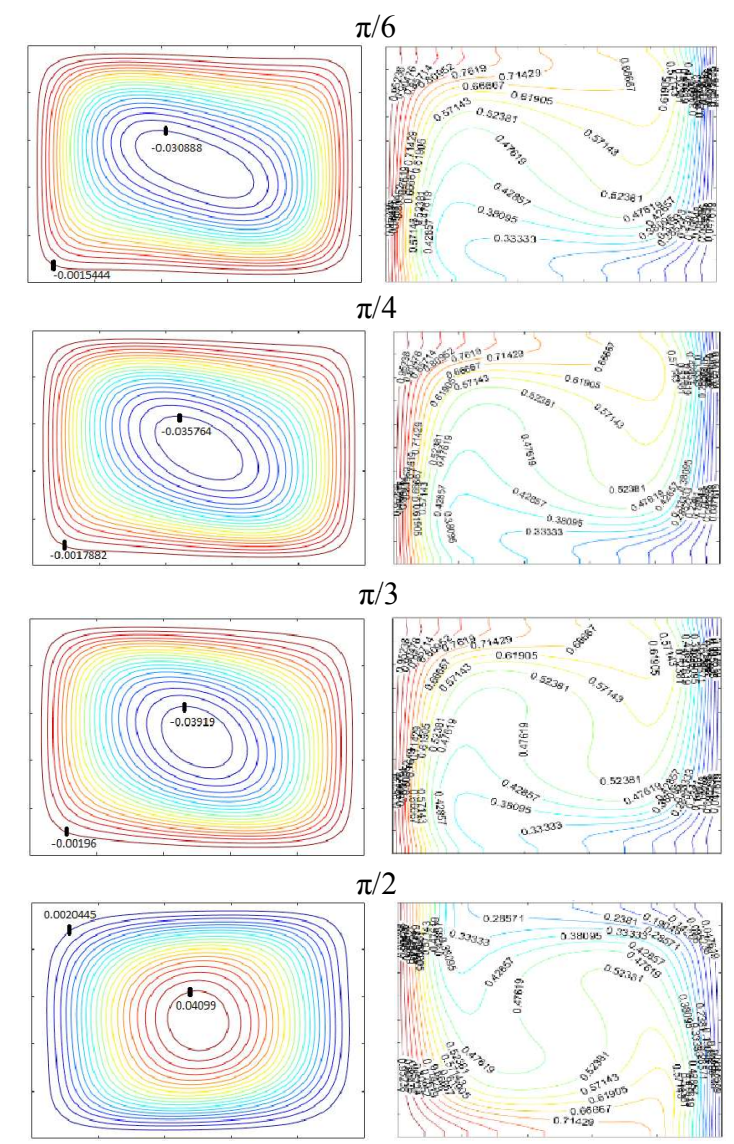

Fig. 8. Streamlines contours and isotherms at various angles of inclination $\left(\mathrm{Gr}=10^{4}, \phi=10 \%, \operatorname{Pr}=6.2\right)$.

To analyze the effect of inclination on stream function and temperatures, the case of a nanofluid with a volume fraction of $10 \%$ was adopted. Fig. 7 shows that increasing the angle of inclination produces a change in the stream function and temperature profiles. Indeed, we can notice that the increase of the inclination has a direct effect on the intensification of the contours and on the profiles of temperature, where we can notice the migration of the temperature isolines from the hot source towards the cold source

\section{Conclusion}

In this paper, a code based on a finite difference method using the S.O.R iteration and the upwind system was developed. Several numerical simulations have been performed to validate the code, comparing the results with previous works. The good agreement of the results of the code was thus confirmed, which allowed using this code as a prediction tool in the study of the influence of the nanofluid concentration as well as the inclination of the cavity on the thermal performances. An analysis of the results concluded that the improvement in heat transfer is proportional to the increase in the volume fraction of the nanofluid, and that the variation of the inclination affects the thermal enhancement, where the transfer becomes more efficient with tilt of $\pi / 6$.

\section{References}

1. K. Fouladi, A. P. Wemhoff, L. Silva -Llanca, K. Abbasi, and A. Ortega, Appl. Therm. Eng., vol. 124, pp. 929-939, (2017).

2. A. R. Rahmati, A. Rayat Roknabadi, and M. Abbaszadeh, Alexandria Eng. J., vol. 55, no. 4, pp. 3101-3114, (2016).

3. A. Y. Snegirev, Combust. Flame, vol. 136, no. 1-2, pp. 51-71, (2004).

4. A. S. Yang, C. Y. Wen, Y. H. Juan, Y. M. Su, and J. H. Wu, Appl. Therm. Eng., vol. 70, no. 1, pp. 219-230, (2014).

5. A. E. Conradie, J. D. Buys, and D. G. Kröger, Appl. Therm. Eng., vol. 18, no. 97, pp. 25-45, (1998).

6. H. Karatas and T. Derbentli, Int. J. Therm. Sci., vol. 123, pp. 129-139, (2018).

7. M. A. Ismael and H. F. Jasim, Int. J. Mech. Sci., vol. 135, pp. 190-202, (2018).

8. S. Houat and Z. E. Bouayed, Energy Procedia, vol. 139, pp. 186-191, (2017).

9. Y. Wang, G. Qin, W. He, and Z. Bao, Int. J. Heat Mass Transf., vol. 121, pp. 1055-1072, (2018).

10. T. Armaghani, A. Kasaeipoor, N. Alavi, and M. M. Rashidi, J. Mol. Liq., vol. 223, pp. 243-251, (2016)

11. A. Boualit, N. Zeraibi, T. Chergui, M. Lebbi, L. Boutina, and S. Laouar, Int. J. Hydrogen Energy, vol. 42, no. 13, pp. 8611-8623, (2017).

12. K. Khanafer, K. Vafai, and M. Lightstone, Int. J. Heat Mass Transf., vol. 46, no. 19, pp. 36393653, (2003).

13. T. Kogawa, L. Chen, J. Okajima, A. Sakurai, A. Komiya, and S. Maruyama, Appl. Therm. Eng., vol. 131, pp. 141-149, (2018). 